\title{
Diagnostic accuracy of diastolic function evaluation in acute dyspnea
}

\author{
Maria Immacolata Arnone ${ }^{1}$, Alfonso Sforza ${ }^{2}$, Maria Viviana Carlino ${ }^{1}$, Mario Guarino $^{3}$, \\ Fucile Ilaria $^{4}$, Nicola De Luca ${ }^{1}$, and Costantino Mancusi ${ }^{1}$ \\ ${ }^{1}$ Affiliation not available \\ ${ }^{2}$ Cardiology Department and Cardiology Intensive care Unit, Santa Maria delle Grazie, \\ Pozzuoli, Italy \\ ${ }^{3}$ Emergency Department, CTO Hospital \\ ${ }^{4}$ Federico II University Hospital
}

September 25, 2021

\begin{abstract}
Background Acute dyspnea (AD) is one of the main reasons for admission to the Emergency Department (ED). In the last years integrated ultrasound examination (IUE) of lung, heart and inferior vena cava be-come an extension of clinical examination for a fast differential diagnosis. The aim of present study is to assess the feasibility and diagnostic accuracy of diastolic function evaluation for diagnosing acute heart failure (aHF) in patients with acute dyspnea. Methods We included 113 patients presenting to the ED of CTO Hospital in Naples (Italy) for AD. All pa-tients underwent IUE of lung-heart-IVC with a portable ultrasound device. Left ventricle diastolic function was assessed using pulse wave doppler at the tips of the mitral valve and $\mathrm{E}$ wave velocity and E/A ratio were recorded. The FINAL diagnosis determined by two expert reviewers: acute HF or non-acute HF (non-aHF). We used $2 \times 2$ contingency tables to analyze sensitivity, specificity, positive predictive and negative predictive value of ultrasound parameters for the diagnosis of AD, comparing with the FINAL diagnosis. Results Lung ultrasound (LUS) showed high sensitivity, good specificity and accuracy in identification of patients with HF. However the highest accuracy was obtained by diastolic function parameters. The E/A ratio, detected in patients in sinus rhythm, showed the highest diagnostic performance with an AUC for aHF of 0.913. Conclusion In patients presenting with AD E/A ratio is easy to obtain in a fast ultrasound protocol and showed an excellent accuracy for diagnosis of acute HF.
\end{abstract}

\section{Diagnostic accuracy of diastolic function evaluation}

in acute dyspnea

Maria Immacolata Arnone ${ }^{1}$, Alfonso Sforza ${ }^{2}$, Maria Viviana Carlino ${ }^{3}$, Mario Guarino ${ }^{1}$, Riccardo Candido $^{1}$, Ilaria Fucile ${ }^{4}$, Nicola De Luca ${ }^{4}$, Costantino Mancusi ${ }^{*}$

${ }^{1}$ Emergency Department, CTO Hospital, Naples; ${ }^{2}$ Cardiology Department and Cardiology Intensive care Unit, Santa Maria delle Grazie, Pozzuoli, Italy, : ${ }^{3}$ Cardiology Department and Cardiology Intensive care Unit, San Giuliano Hospital, Giugliano, Italy, ${ }_{4}$ Hypertension Research Center \& Department of Advanced Biomedical Science, Federico II University Hospital, Naples, Italy

*Corresponding Author: Costantino Mancusi, Hypertension Research Center \& Department of Advanced Biomedical Science, Federico II University Hospital, Via Sergio Pansini 5, 80131, Naples, Italy. Phone +390817462211 email:costantino.mancusi@unina.it 


\section{Authors' contributions:}

Maria Immacolata Arnone, Alfonso Sforza, Maria Viviana Carlino and Costantino Mancusi had the idea for this article and recruited patients, Maria Immacolata Arnone and Costantino Mancusi performed statistical analysis and drafted the paper and Mario Guarino, Riccardo Candido, Ilaria Fucile, Nicola De Luca, critically revised the work. All authors read and approved the final version of the manuscript.

Key Words: Lung ultrasound, focused cardiac ultrasonography, multi-organ ultrasound, diastolic function, Emergency department.

\section{Abstract \\ Background}

Acute dyspnea (AD) is one of the main reasons for admission to the Emergency Department (ED). In the last years integrated ultrasound examination (IUE) of lung, heart and inferior vena cava become an extension of clinical examination for a fast differential diagnosis. The aim of present study is to assess the feasibility and diagnostic accuracy of diastolic function evaluation for diagnosing acute heart failure (aHF) in patients with acute dyspnea.

\section{Methods}

We included 113 patients presenting to the ED of CTO Hospital in Naples (Italy) for AD. All patients underwent IUE of lung-heart-IVC with a portable ultrasound device. Left ventricle diastolic function was assessed using pulse wave doppler at the tips of the mitral valve and $\mathrm{E}$ wave velocity and $\mathrm{E} / \mathrm{A}$ ratio were recorded. The FINAL diagnosis determined by two expert reviewers: acute HF or non-acute HF (non-aHF). We used $2 \times 2$ contingency tables to analyze sensitivity, specificity, positive predictive and negative predictive value of ultrasound parameters for the diagnosis of $\mathrm{AD}$, comparing with the FINAL diagnosis.

\section{Results}

Lung ultrasound (LUS) showed high sensitivity, good specificity and accuracy in identification of patients with HF. However the highest accuracy was obtained by diastolic function parameters. The E/A ratio, detected in patients in sinus rhythm, showed the highest diagnostic performance with an AUC for aHF of 0.913 .

\section{Conclusion}

In patients presenting with $\mathrm{AD} \mathrm{E} / \mathrm{A}$ ratio is easy to obtain in a fast ultrasound protocol and showed an excellent accuracy for diagnosis of acute HF.

\section{INTRODUCTION}

Initial assessment of patients with acute dyspnea is commonly based on medical history, physical examination, chest X ray, 12 leads electrocardiogram, arterial blood gas analysis and routine blood tests .

In the last years integrated ultrasound examination (IUE) of lung, heart and inferior vena cava become an extension of clinical examination for a fast assessment of acute dyspnea in emergency setting .

Lung ultrasound (LUS) is widely demonstrated to be superior for the identification and evaluation of extravascular lung water when compared to chest X ray . The implementation of LUS with clinical assessment improves the diagnostic accuracy for acute HF in the context of emergence department. Focused cardiac ultrasonography (FoCUS) is a non-invasive and reproducible examination that has a great diagnostic and prognostic value in patients with $\mathrm{HF}$.

We have recently demonstrated that the combination of positive LUS with FoCUS, including dilated left atrium and reduced ejection fraction, substantially extends the spectrum of recognizable acute HF .

Left ventricle diastolic dysfunction, represent the main pathophysiological abnormalities in HF as it plays an important role in determining increased LV filling pressures with consequent development of venous 
congestion and pulmonary edema. Its assessment in the context of emergency department is feasible and may help to improve rapid diagnostic assessment of patients with acute dyspnea .

The aim of present study is to assess feasibility and the diagnostic accuracy of diastolic function assessment included in a fast protocol of IUE for diagnosing acute HF in patients with undifferentiated dyspnea in emergency department (ED).

\section{MATERIALS AND METHODS:}

From January 2019 to June 2019 we enrolled 113 patients presenting to the ED of CTO Hospital in Naples (Italy) for acute dyspnea or sudden worsening of chronic dyspnea within the previous 48 hours. Patients with dyspnea of traumatic origin were excluded. All patients underwent clinical examination, blood gas analysis, chest X-ray, ECG, and routine blood tests (including N-terminal pro-BNP (NT-pro-BNP). NT-pro- BNP was considered negative if $<300 \mathrm{pg} / \mathrm{mL}$ and in favour of acute decompensated $\mathrm{HF}$ if $>1800 \mathrm{pg} / \mathrm{mL}$ in patients older than 75 years, $900 \mathrm{pg} / \mathrm{mL}$ in patients from 50 to 75 years old, $450 \mathrm{pg} / \mathrm{mL}$ in patients younger than 50 years. Chest X-ray was considered positive when detected interstitial syndrome and/or the presence of pleural effusion. All patients underwent IUE with a portable ultrasound device using convex probe for LUS and cardiac probe for heart and inferior vena cava evaluation. An emergency physician with good experience of LUS and transthoracic echocardiography, who was not taking care of the patient, performed IUE with the patient in semi-sitting or supine position. For LUS was followed a simplified protocol that provides two scans at each side, anteriorly on the II intercostal space, mid-clavicular line, and lateral on the V intercostal space, mid-axillary line, to sample upper and lower lungs . The presence or the absence of interstitial syndrome (IS, defined as the presence of at least $3 \mathrm{~B}$ lines for lung field) and the presence or the absence of pleural effusion (defined as hypo-anechoic space between the parietal and visceral pleura) was evaluated. Lung ultrasound was defined positive for bilateral IS and/or effusion if IS and/or effusion was present at least in the lateral scan (V intercostal space, mid-axillary line) bilaterally. FoCUS examination included two projections (parasternal long-axis view and apical view). Ejection fraction (EF) was estimated visually and categorized as preserved if $>40 \%$ or reduced if [?] $40 \%$, based on cut point provided by the ESC guidelines . Left atrium was considered dilated if anteroposterior diameter (in the parasternal long-axis view) was visually estimated to be $>4 \mathrm{~cm}$ in both genders. The IVC was evaluated in subcostal view for the presence of dilatation (visually estimated to be $>2 \mathrm{~cm}$ ) and hypo-reactivity with breathing (variation of size $<50 \%$ about). LV diastolic function was evaluated using pulse wave doppler at the tips of the opened mitral valve in 4- chambers apical view and $\mathrm{E}$ wave velocity and $\mathrm{E} / \mathrm{A}$ ratio were recorded. If the patient was in sinus rhythm three consecutive cycles at end expiration were recorded, and the average of these three E-waves was registered. If the patient was presenting with an irregular rhythm, such as atrial fibrillation, five consecutive cycles and the average of these five E-waves were registered .

The duration of ultrasound examination was always $<3$ minutes, and it was done within 30 minutes from the arrival of the patients in ED. The final diagnosis, considered as the gold standard, was issued by two independent observers who had access to the entire medical chart (from Emergency Department admission to hospital discharge) of each patient (medical history, clinical examination, blood gas analysis, CXR, ECG, and routine blood tests (including NT-pro- BNP). Based on this revision, patients were classified into two groups: acute HF or non-cardiac dyspnea (non-AHF). Acute HF was defined according to current guidelines . In patients with coexistence of heart failure and another cause of dyspnea, the main diagnosis was acute HF . Informed consent was obtained from each patient included in the study and follows the principles of the Declaration of Helsinki. The study was approved by the ethics committee of the University of Naples Federico II. Data were analysed using SPSS version 24.0 (SPSS, Chicago, IL, USA). Continuous data are expressed as mean +- 1 standard deviation and categorical variables as percentages. Quantitative variables were compared by using Student's $t$-test, while chi-square distribution was used to compare categorical variables. A $P$-value $<.05$ was considered statistically significant. The population was divided into patients acute HF or non-cardiac dyspnea (non-AHF). The performance of each different diagnostic tests for the diagnosis of acute HF (chest x-ray, NT-pro- BNP, bilateral IS and/or effusion, dilated left atrium, and EF [?] 40\%, $\mathrm{E} / \mathrm{A}$ ratio and $\mathrm{E}$ wave) was analysed, and comparisons were made among them, using sensitivity, specificity, 
positive predictive value, negative predictive value. Confidence intervals (CI) at $95 \%$ were calculated for sensitivity, specificity, positive predictive value, and negative predictive value. Areas under the curve (AUC) and receiver operating characteristic (ROC) curve were used to compare the performance of each different ultrasound diagnostic tests in relation to the final diagnosis. Yuden index was used to calculate the optimal threshold value (cut-off point) for $\mathrm{E}$ wave and $\mathrm{E} / \mathrm{A}$ ratio to identify patients with acute HF.

\section{RESULTS}

Our study population included 113 patients. 36 patients (31.9\%) had acute HF, and 77 patients (68.14\%) had non-cardiac dyspnea. 21 patients had atrial fibrillation. Table 1 displays the final diagnosis of patients obtained after the review of all clinical folder by two independent observers. Table 2 shows the baseline characteristics of the two subgroups based on the final diagnosis. Patients with acute HF had higher systolic blood pressure, NT-pro- BNP, and creatinine levels than patients with non-cardiac dyspnea (Table 1, all $p$ $<.05)$. Patients with acute HF had more frequent medical history of heart failure and atrial fibrillation. In contrast patients with non-cardiac dyspnea had higher C-reactive protein and procalcitonin levels.

Table 3 shows diagnostic performance of all ultrasound parameters together with chest X ray and NTpro BNP for the diagnosis of acute HF. Chest X ray and NT-pro- BNP had the lowest accuracy for the identification of acute HF ( $65.77 \%$ and $71.28 \%$ respectively). LUS positivity for bilateral IS and/or effusion exhibited high sensitivity $(86.11 \%)$, good specificity $(71.43 \%)$ and accuracy for acute HF $(76.11 \%)$. Dilated left atrium showed the highest sensibility in identifying acute HF (94.29\%), NT-pro BNP showed the highest specificity (98.31\%). In an additional analysis we calculate, through the Yuden Index, the best cut off value for $\mathrm{E}$ wave velocity and $\mathrm{E} / \mathrm{A}$ ratio to identify acute $\mathrm{HF}$. The Yuden Index value for $\mathrm{E}$ wave velocity was $72.50 \mathrm{~cm} / \mathrm{sec}$, the best cut off value for $\mathrm{E} / \mathrm{A}$ ratio was 0.95 . Table 4 shows diagnostic performance of the $\mathrm{E}$ wave and E/A ratio best cut off values. Both diastolic parameters had high specificity in identifying acute HF (92,54\% and $91.04 \%$ respectively).

Figure 1 shows the area under the ROC curve (AUC) of single parameters in a subgroup of patients in sinus rhythm $(n=92)$. The best cut off value of $\mathrm{E} / \mathrm{A}$ ratio showed the highest diagnostic performance with an AUC in identifying HF of 0.91 . The ROC curve comparing accuracy of different ultrasound modalities showed that E/A ratio had higher diagnostic accuracy than LUS positive (0.75) and a tendency to be more statistically significant than dilated left atrium (all $P<.01$ ).

\section{DISCUSSION:}

The present study demonstrated that the evaluation of diastolic dysfunction in a fast-integrated ultrasound protocol has an excellent accuracy for diagnosis of acute HF in patients presented to the ED with acute dyspnea. Both E wave velocity and E/A ratio are easy to obtain within a 3 minutes fast ultrasound protocol and allows better discrimination of acute HF among patients with acute dyspnea.

The diagnosis of $\mathrm{HF}$ in patients presenting to the $\mathrm{ED}$ with acute dyspnea remains challenging. It was demonstrated the utility of integrated heart and lung ultrasound examination for an ultrafast diagnosis of HF in the emergency setting. The main pathophysiological determinant dyspnea in acute HF is increased pulmonary wedge pressure which causes lung interstitial and alveolar edema. LUS is accurate in diagnosing alveolar- interstitial syndrome. The B lines pattern that configures the framework of an alveolar-interstitial syndrome may reflect the presence of either cardiogenic or inflammatory edema, or fibrosis . Pleural effusion may instead be the result of inflammation of the lung parenchyma or heart failure with increased central venous pressure. For this reason the presence of bilateral IS/effusion alone has high sensitivity as already demonstrated in previous studies but a suboptimal specificity as also observed in the present study.

FoCUS identifies cardiac abnormalities and can help to explain the acute onset of HF. Diastolic dysfunction of the left ventricle is presented in all types of HF regardless of the value of EF. It represents a combination of impaired left ventricular (LV) relaxation, restoration forces, myocyte lengthening load, and atrial function, culminating in increased LV filling pressures with congestion or pulmonary edema and left atrial dilation . We have recently demonstrated that left atrial dilatation has an excellent accuracy for the identification of 
patients with acute HF when combined with LUS exhibiting wet lungs. However LA dilatation also reflect a chronic diastolic dysfunction, so alone has a good sensitivity but suboptimal specificity.

Current Doppler echocardiography guidelines recommend using early to late diastolic trans mitral flow velocity (E/A) to assess diastolic function, and $\mathrm{E}$ to early diastolic mitral annular tissue velocity (E/e') to estimate LV filling pressures. In our study the $\mathrm{E}$ wave velocity showed an excellent accuracy in diagnosing and excluding acute HF when compared with other lung and heart ultrasound parameters. E wave velocity can be achieved in all patients, allowing rapid evaluation also in patients with atrial fibrillation. Moreover in subgroup of patients with sinus rhythm, in which is possible to measure $\mathrm{E}$ and $\mathrm{A}$ wave velocity, $\mathrm{E} / \mathrm{A}$ ratio showed the best diagnostic accuracy. Higher E wave velocity and E/A ratio are associated with higher LV filling pressure which is the main determinant of development of pulmonary venous congestion and acute HF . In our study E/A ratio showed high specificity and tendency to be more statistically significant then dilated left atrium allowing to extend the spectrum of recognizable acute HF also in patients without dilated left atrium.

In this study the operator was experienced in both FoCUS and LUS. It was demonstrated that ED were able to perform and interpret focused echocardiography reliably after a short duration of training . T. Saul and colleagues showed that assessment of diastolic function is easy to obtain from emergency physicians credentialed in FoCUS with very good inter-rater Reliability .

Our study has some limitations. First the population sample is limited. A larger study population is need to confirm our results and the tendency of E/A ratio to be statistically more significant than dilated left atrium. The operator in this study was experienced in both echo and LUS. Nevertheless, echo and LUS, can both be learned with a steep learning curve. The left ventricle diastolic function evaluation with pulse Doppler imaging has been proven to be feasible and especially useful for diagnosis . Thus a protocol could probably be implemented into an ED setting after consideration of sufficient training and quality assessment

\section{CONCLUSIONS}

Integrated ultrasound examination is a useful tool for differential diagnosis in patients admitted to the ED with acute dyspnoea. In all type of heart failure regardless ejection fraction, the left ventricle diastolic dysfunction plays an important role in congestion or pulmonary oedema and consequent respiratory symptoms. $\mathrm{E}$ wave velocity and $\mathrm{E} / \mathrm{A}$ ratio showed an excellent diagnostic accuracy in diagnosing acute HF when assessed in ultrafast ultrasound protocol.

Conflicts of interest: none

Funding: none

Acknowledgements: NONE

TABLE 1 FINAL diagnosis by the two independent observers

\begin{tabular}{ll}
\hline Final diagnosis & Cases \\
\hline Acute heart failure & $36(31.9 \%)$ \\
Pneumonia & $16(14.2 \%)$ \\
Acute or exacerbation of COPD/asthma & $27(23.9 \%)$ \\
Pleural effusion & $11(9.7 \%)$ \\
Pulmonary embolism & $1(0.9 \%)$ \\
Primary or secondary lung neoplasm & $14(12.4 \%)$ \\
Pulmonary fibrosis & $2(1.8 \%)$ \\
Anaemia & $3(2.7 \%)$ \\
Asma & $2(1.8 \%)$ \\
Anxiety & $1(0.9 \%)$
\end{tabular}




\begin{tabular}{ll}
\hline Final diagnosis & Cases \\
\hline Identification of acute heart failure & $36(31.9 \%)$ \\
Acute heart failure & $77(68.14 \%)$ \\
Non-cardiac dyspnea & \\
\hline
\end{tabular}

TABLE 2 Baseline characteristics and clinical findings detected at the time of patient presentation in the Emergency Department

\begin{tabular}{|c|c|c|c|}
\hline & $\begin{array}{l}\text { Acute heart failure }(\mathrm{n}= \\
36)\end{array}$ & $\begin{array}{l}\text { Noncardiac dyspnea }(\mathrm{n}= \\
77)\end{array}$ & $P$ \\
\hline Age (years) & $73.9 \pm 12.6$ & $69.35 \pm 13.5$ & .90 \\
\hline Sex (female) & $27.7 \%$ & $39 \%$ & .247 \\
\hline $\begin{array}{l}\text { Medical history of } \\
\text { chronic obstructive } \\
\text { pulmonary disease }\end{array}$ & $41.7 \%$ & $42.9 \%$ & .905 \\
\hline $\begin{array}{l}\text { Medical history of heart } \\
\text { failure and/ or ischemic } \\
\text { heart disease }\end{array}$ & $47.2 \%$ & $6.5 \%$ & .000 \\
\hline Medical history of FA & $30.6 \%$ & $13 \%$ & 0.25 \\
\hline Heart rate $(\mathrm{bpm})$ & $85.5 \pm 23.5$ & $88.7 \pm 17$ & .416 \\
\hline $\begin{array}{l}\text { Respiratory rate } \\
\text { (breaths/min) }\end{array}$ & $22.9 \pm 6.9$ & $22.3 \pm 7.6$ & .745 \\
\hline Systolic BP (mm Hg) & $149 \pm 28.2$ & $144 \pm 24.3$ & .356 \\
\hline Diastolic BP (mm Hg) & $82 \pm 18.8$ & $82 \pm 16$ & .968 \\
\hline $\begin{array}{l}\text { Serum creatinine } \\
(\mathrm{mg} / \mathrm{dL})\end{array}$ & $1.47 \pm 0.77$ & $0.96 \pm 0.44$ & .0001 \\
\hline NT-pro-BNP & $1115 \pm 883$ & $169.69 \pm 264.4$ & .0001 \\
\hline $\begin{array}{l}\text { White blood cell count } \\
\left(\times 10^{3} / \mu \mathrm{L}\right)\end{array}$ & $9.45 \pm 4.45$ & $11.23 \pm 4.45$ & .052 \\
\hline Oxygen saturation (\%) & $93.11 \pm 6.1$ & $92.2 \pm 6.3$ & .947 \\
\hline $\mathrm{PaO} 2 / \mathrm{FiO} 2$ & $291.37 \pm 81.26$ & $273.58 \pm 84.48$ & .348 \\
\hline $\mathrm{pH}$ & $7.41 \pm 0.072$ & $7.43 \pm 0.075$ & .103 \\
\hline Lactate level $(\mathrm{mmol} / \mathrm{L})$ & $1.66 \pm 1.10$ & $1.76 \pm 1.12$ & .672 \\
\hline Protein $\mathrm{C}$ reactive & $38.74 \pm 62.07$ & $55.78 \pm 88.51$ & .308 \\
\hline Procalcitonin & $0.48 \pm 1.41$ & $2.02 \pm 10.2$ & .458 \\
\hline
\end{tabular}

TABLE 3 Sensitivity, specificity, positive and negative predictive value and accuracy of the main diagnostic features

\begin{tabular}{llllll}
\hline Parameter & $\begin{array}{l}\text { Sensitivity }(\%) \\
(95 \% \text { CI })\end{array}$ & $\begin{array}{l}\text { Specificity }(\%) \\
(95 \% \text { CI })\end{array}$ & $\begin{array}{l}\text { PPV }(\%)(95 \% \\
\text { CI })\end{array}$ & $\begin{array}{l}\text { NPV }(\%)(95 \% \\
\text { CI })\end{array}$ & Accuracy $(\%)$ \\
\hline NT-pro-BNP & $25.71(12.49-$ & 98.31 & $90.00(54.34-$ & $69.05(64.67-$ & 71.28 \\
& $43.26)$ & $(90.91-99.96)$ & $98.55)$ & $73.11)$ &
\end{tabular}




\begin{tabular}{llllll}
\hline Parameter & $\begin{array}{l}\text { Sensitivity }(\%) \\
(95 \% \mathrm{CI})\end{array}$ & $\begin{array}{l}\text { Specificity }(\%) \\
(95 \% \mathrm{CI})\end{array}$ & $\begin{array}{l}\text { PPV }(\%)(95 \% \\
\text { CI })\end{array}$ & $\begin{array}{l}\text { NPV }(\%)(95 \% \\
\text { CI })\end{array}$ & Accuracy $(\%)$ \\
\hline Chest x-ray & $38.89(23.14-$ & $78.67(67.68-$ & $46.67(32.50-$ & $72.84(66.83-$ & 65.77 \\
& $56.54)$ & $87.29)$ & $61.39)$ & $78.12)$ & \\
Dilated left & $94.29(80.84-$ & 64.86 & $55.93(47.96-$ & 96.00 & 74.31 \\
atrium & $99.30)$ & $(52.89-75.61)$ & $63.61)$ & $(86.08-98.94)$ & \\
Bilateral IS & $86.11(70.50-$ & $71.43(60.00-$ & 58.49 & 91.67 & 76.11 \\
and/or effusion & $95.33)$ & $81.15)$ & $(49.16-67.25)$ & $(82.81-96.17)$ & \\
EF [?] 40 & 47.22 & 93.24 & 77.27 & 78.41 & 78.18 \\
& $(30.41-64.51)$ & $(84.93-97.77)$ & $(57.68-89.45)$ & $(72.60-83.27)$ & \\
\hline
\end{tabular}

TABLE 4 Sensitivity, specificity, positive and negative predictive value and accuracy of E wave and E/A ratio best cut off values

\begin{tabular}{llllll}
\hline Parameter & $\begin{array}{l}\text { Sensitivity }(\%) \\
(95 \% \mathrm{CI})\end{array}$ & $\begin{array}{l}\text { Specificity }(\%) \\
(95 \% \mathrm{CI})\end{array}$ & $\begin{array}{l}\text { PPV }(\%)(95 \% \\
\mathrm{CI})\end{array}$ & $\begin{array}{l}\text { NPV }(\%) \\
(95 \% \mathrm{CI})\end{array}$ & Accuracy $(\%)$ \\
\hline E wave velocity & $86.11(70.50-$ & 92.54 & $70.59(48.47-$ & 82.67 & 80.43 \\
& $95.33)$ & $(83.44-97.53)$ & $85.96)$ & $(76.49-87.49)$ & \\
E/A ratio & $88 \%(68.78-$ & $91.04 \%(81.5-$ & $78.57 \%(62.77-$ & $95.31 \%(87.52-$ & 90.22 \\
& $97.45)$ & $96.64)$ & $88.86)$ & $98.33)$ & \\
\hline
\end{tabular}

\section{Figure legend.}

Figure 1. Receiver operating characteristic (ROC) curve comparing accuracy of different ultrasound modalities. 


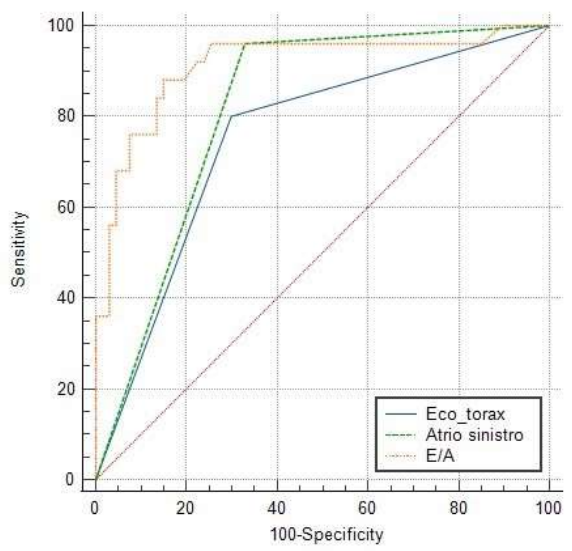

\begin{tabular}{llll} 
Parameter & $\begin{array}{l}\text { Area under } \\
\text { curve (AUC) }\end{array}$ & $\mathbf{9 5 \%} \mathrm{Cl}$ & $\mathbf{p}$ \\
\hline E/A ratio & 0,913 & $0,836-0,962$ & - \\
Dilated left atrium & 0,816 & $0,721-0,889$ & $0.05^{*}$ \\
Bilateral IS and/or effusion & 0,751 & $0,650-0,835$ & $0.0010^{*}$
\end{tabular}

Legend. Cl: Confidence Interval, IS: interstitial syndrome

*p vs E/A ratio

Figure 1. Receiver operating characteristic (ROC) curve comparing accuracy of different ultrasound modalities. 